\title{
Effects of propolis extract on growth of Entamoeba histolytica
} (trophozoites) in vitro

تأثير المستخلص الكحولي للعكبر على نمو الأطوار المتظذية للأميبا الحالة للنسج في الزجاج

Naksheen M.Ardalan Ekhlas mushref Zahra'a Abdul-Raheem Ahmed

Dept. of Biology/ College of Science for Women/ University of Baghdad

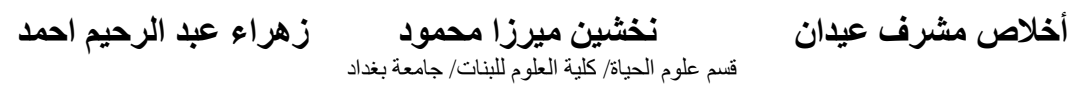

Abstract

Amoebiasis is the third leading cause of death from parasitic disease worldwide. The causative protozoan parasite, Entamoeba histolytica, is a potent pathogen. Propolis is a natural resinous substance collected by bees from vegetable sources and it has a therapeutic properties have been investigated in this work. So we evaluated the inhibitory activity of ethanolic extracts of propolis (EEP) on Entamoeba histolytica trophozoite growth. Propolis inhibited the growth of E.histolytica trophozoites and the level of inhibition varied according to the extract concentration and incubation times, it also showed a marked activity on cell lysis of trophozoite. The highest reduction of parasite growth was observed in culture exposed to $(25,50 \mathrm{mg} / \mathrm{ml})$ of propolis, in all incubation periods $(24,48$ )h. Growth reduction by $76 \%$ was observed in $12.5 \mathrm{mg} / \mathrm{ml}$ propolis-treated culture, while the concentrations of $(25,50) \mathrm{mg} / \mathrm{ml}$ were able to inhibit growth by more than $90 \%$. Light microscopic study showed morphological changes in

E.histolytica trophozoites.

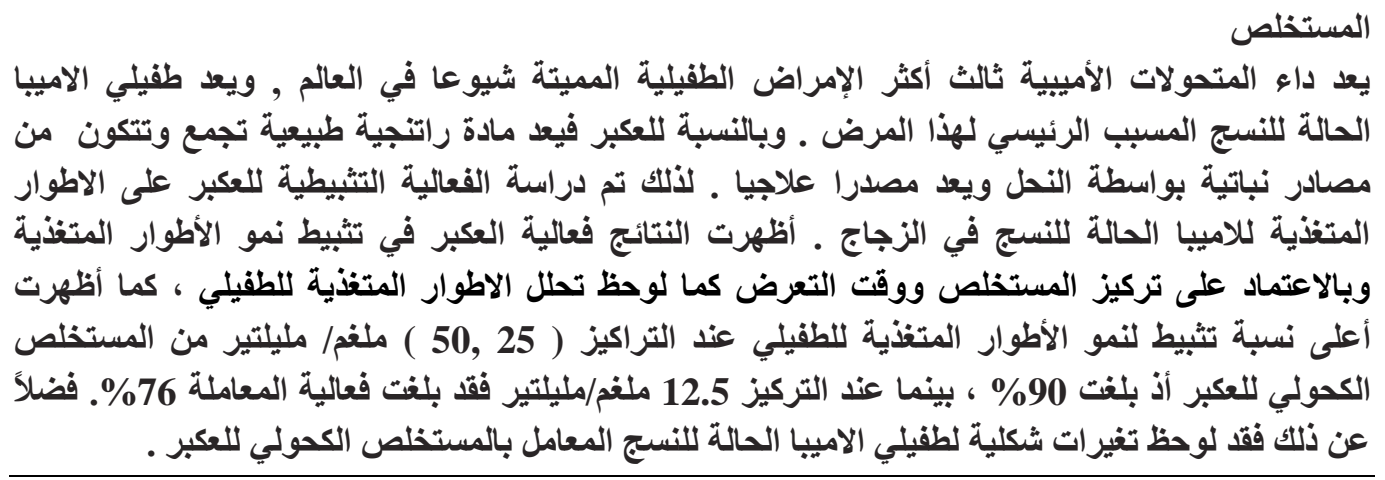

\section{Introduction}

Amoebiasis is one of the major health problems in several developing countries; as a result amoebic dysentery is common in tropic and subtropics regions [1]. Secreting proteinases that dissolve host tissues, killing host cells on contact, and engulfing red blood cells, E histolytica trophozoites invade the intestinal mucosa; causing amoebic colitis is acquired by ingestion of Entamoeba histolytica cysts [2]. Although cysts are the infective stage, have not been induced in vitro, due to the absence of a medium or method that supports the in vitro mass encystation of this species [3]. This has been a barrier to research to control methods for this phase of E. histolytica life cycle. Metronidazole is known to be highly effective amoebicide and is considered to be the drug of choice for the treating amoebiasis, but this drug has been found to noccose 
mutagenic effects in bacteria and is carcinogenic in some animals [4]. In addition, this drug has several adverse effects for which the most common are gastrointestinal disturbances, especially nausea [5]. In vitro evaluation of natural remedies from propolis of various plant species are required to prove their claimed effectiveness against Entamoeba histolytica, the causal agent of the disease and also for a possible drug development.

Propolis is a complex resinous material produced by honeybees from plant exudates, beeswax, and bee secretions [6] and has protective function on honeycombs, especially against microrganisms [7], Propolis is a sticky dark-colored material that honey bees collect from plants. The chemical composition of propolis is very complex and is dependent upon the plant source. But exudates of different poplar species are the main sources of propolis in the temperate zone, including Europe, Asia and North America. Samples originating from these regions are characterized by similar chemical composition; the most important constituents appeared to be phenolics: flavonoids, aromatic acids, caffeic acid and its esters, cinnamic acids [8,9] propolis is composed of resin and vegetable balsam 50\%, wax 30\%, aromatic oils 10\%, pollen $5 \%$ and various other substances depending upon the vegetation of the area $[10,11]$. Propolis is known for antimicrobial, anti-oxidant and antitumoral properties [12]. In addition, propolis ethanolic preparation shows in vitro anti-microbial activity mainly against Gram positive (Staphylococci and Strepthococci spp.) and Gram-negative bacteria (E. coli, K. pneumoniae, P. vulgaris and $P$. aeruginosa, fungi (Candida albicans) and viruses (HIV, Herpes viruses or influenza viruses) [13]. Studies have shown propolis in vitro activity against protozoa, inhibiting proliferation of Toxoplasma gondii and Trichomonas vaginalis [14], Trypanosoma cruzi $[15,9,16]$ and G. duodenalis [17].

The present study was carried out aiming to evaluate the in vitro effects of ethanolic extract of propolis on the growth and morphological change of Entamoeba histolytica trophozoites.

\section{Materials and methods}

\section{Parasites and culture}

E.histolytica trophozoites were xenically cultivated in Locke's egg (LE) medium modified by Boeck and Drobohlav (1925). The strain was isolated from patient at the Al-yarmuk hospital Laboratory in Baghdad.

Trophozoites harvested in log-phase growth within 48-72 h postinoculation. Cells were counted in a haemocytometer (Neubauer cell-counter chamber) and used to study the effects of propolis on cell growth of E. histolytica trophozoites [18] .

\section{Ethanolic Extract of Propolis (EEP)}

The ethanolic extracts of propolis (EEP) were prepared by using a modified technique described by [19]. A crude sample of propolis was collected from Turkey, EEP were obtained treating $15 \mathrm{~g}$ crude propolis in $100 \mathrm{ml}$ of $70 \%$ ethanol, and extracted at room temperature for $24 \mathrm{~h}$. The solution was filtered with Whatman paper number -1 , and placed in amber flasks. Each solution was dried and the residue weighted to prepare stock solution in ethanol at concentration of 5\%. Parasite was treated with different 
concentrations of propolis $(12.5,25,50) \mathrm{mg} / \mathrm{ml}$ for $(24,48) \mathrm{h}$ at $37^{\circ}$ and parasite number and morphology were determined using a Neubauer haemocytometer [20].

\section{Growth inhibition assay}

In order to evaluate the propolis effect on the growth of E.histolytica, $0.02 \times 10^{6}$ trophozoites were incubated in LE medium containing propolis in different concentrations $(12.5,25,50) \mathrm{mg} / \mathrm{ml}$ for $(24,48) \mathrm{h}$ at $37^{\circ}$. In addition, controls were included in all assays (cultures containing only the parasites). After incubation the population density of cultures was estimated with Haemocytometer. For each propolis concentration and controls, five tubes were screened and the cumulative mean and standard deviations were calculated.

\section{Mortality rate}

Growth rate of the parasite tested against propolis was calculated from the trophozoite count per ml, mortality rate of E. hitolytica with respect to propolis at various concentrations was obtained as follow [21]:

$$
\text { Mortality rate }(\%)=\frac{\text { Count } / \mathrm{ml} \text { treated }}{\text { Count } / \mathrm{ml} \text { (untreated control) }} 100-100
$$

\section{The reagent of flavonol content}

The analysis of the flavonol was performed as described in a study [22] by preparation two solutions the first solution preparation by dissolving $10 \mathrm{gm}$ of dry powder of propolis in $5 \mathrm{ml}$ of ethanol alcohol $95 \%$, and the second solution preparation from $10 \mathrm{ml}$ of ethanol alcohol $50 \%$ with $10 \mathrm{ml}$ of potassium hydroxide solution $50 \%$. After mixture of these two solution appearing of yellow color is guide to the flavones.

\section{Statistical analysis}

Analysis of variance was employed in order to evaluate propolis activity on the growth of E. histolytica trophozoites, according to extract concentration and time of incubation. $f$-test and Duncan test was used to determine $P$-values for the differences observed between the test sample and the control. A $P$ - value of 0.05 or less was considered indicative of a statistically significant difference.

\section{Results}

\section{Propolis chemical composition}

The chemical analysis of our propolis sample revealed that its main components are flavones.

\section{Growth inhibition}

The effects of several concentrations of our propolis sample on in vitro growth of $E$. histolytica are summarized in Figs (1), Propolis inhibited the growth of trophozoites and the level of inhibition varied according to the extract concentration and incubation times. Propolis effect was observed with $12.5 \mathrm{mg} / \mathrm{ml}$, but the highest reduction on parasite growth was observed in cultures exposed to $25,50 \mathrm{mg} / \mathrm{ml}$ of extract in all incubation periods. On the other hand, propolis had an effective activity against $E$. histolytica trophozoites at the concentrations of $12.5,25$ and $50 \mathrm{mg} / \mathrm{ml}$, significantly different $(\mathrm{p}<0.05)$ from non-treated (control). 
Growth reduction by $76 \%$ was observed in $12.5 \mathrm{mg} / \mathrm{ml}$ propolis-treated cultures, while the concentrations of 25 and $50 \mathrm{mg} / \mathrm{ml}$ were able to inhibit growth by more than $90 \%$ of trophozoites growth. Treatment of cultures with propolis at $50 \mathrm{mg} / \mathrm{ml}$ inhibited growth by $96 \%$, after 48 -h incubation .

\section{Morphological change}

Besides propolis effect on growth of E.histolytica, light microscope observations, after exposure to propolis at $(12.5,25,50) \mathrm{mg} / \mathrm{ml}$ concentrations and $(24,48) \mathrm{h}$ incubation revealed morphological changes in the cell wall of trophozoites, These changes were not detected in the untreated cultures Figure (2). These morphological and cytoplasmic changes in E.histolytica were in Figure (3).

Also the result showed there was a morphological change and bioprocesses were stopped in E.histolytica treated with EEP, there was a different stage of replication in a number of E.histolytica so the EEP act like colchicine by inhibition the replication as shown in Figure (4).

A few number of E.histolytica cyst (young and diploid nuclei cyst) was found in some cases but no tetra nuclei cyst was found as shown before, Figure (5).

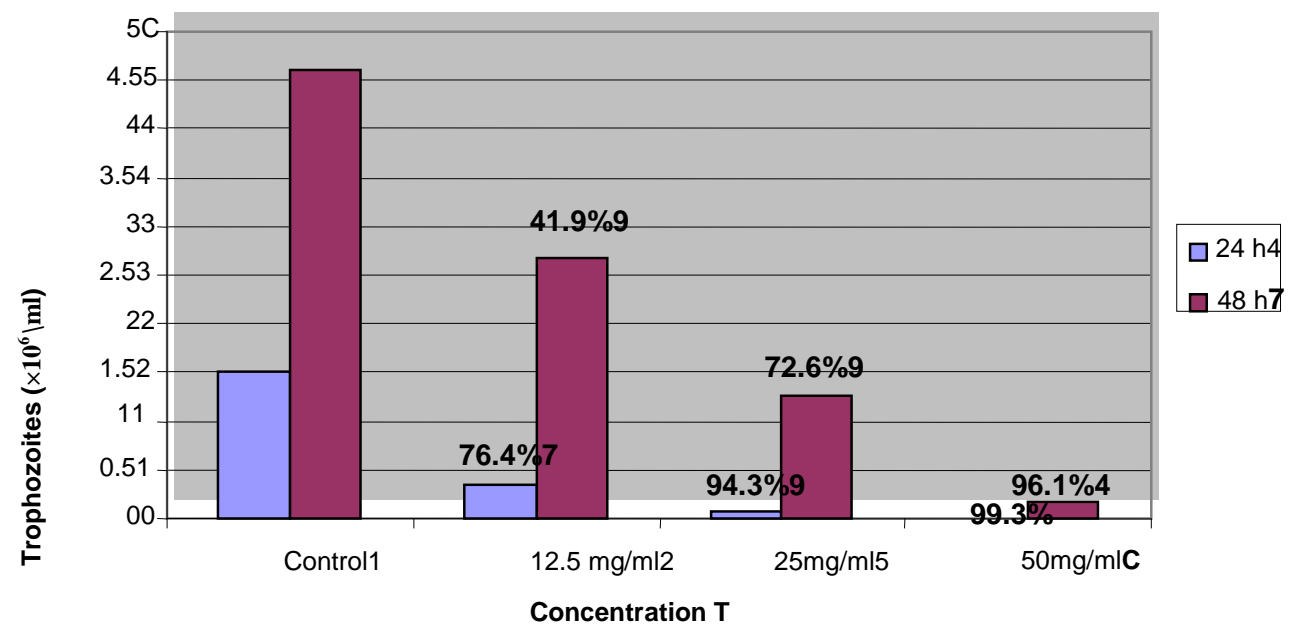

Fig (1): In vitro affects of different concentrations of EEP on the growth of $E$. histolytica trophozoites, after incubation for 24 and $48 \mathrm{~h}$. Data expressed as means of trophozoites number $\left(\mathbf{1 0}^{6}\right) \pm$ standard deviation (SD).

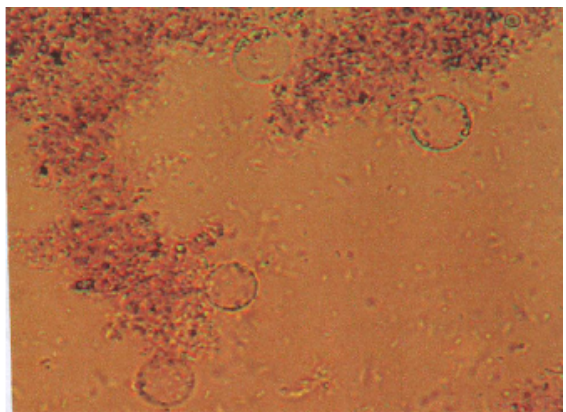

Fig (2): Cultivation of E.histolytica in LE culture media / control (100X).

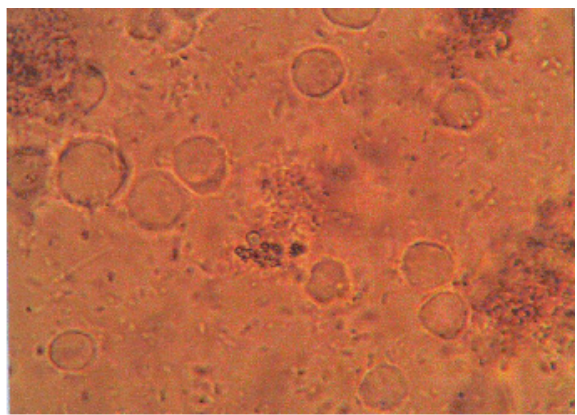

Fig (3): Light microscope observations showed the morphological changes in E.histolytica (100X). 


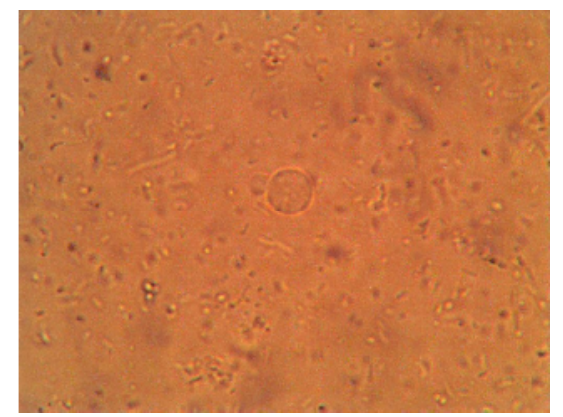

Fig (4): E.histolytica in culture media that treated with EEP showed the inhibition of replication cycle in parasite $(\mathbf{1 0 0 X})$.
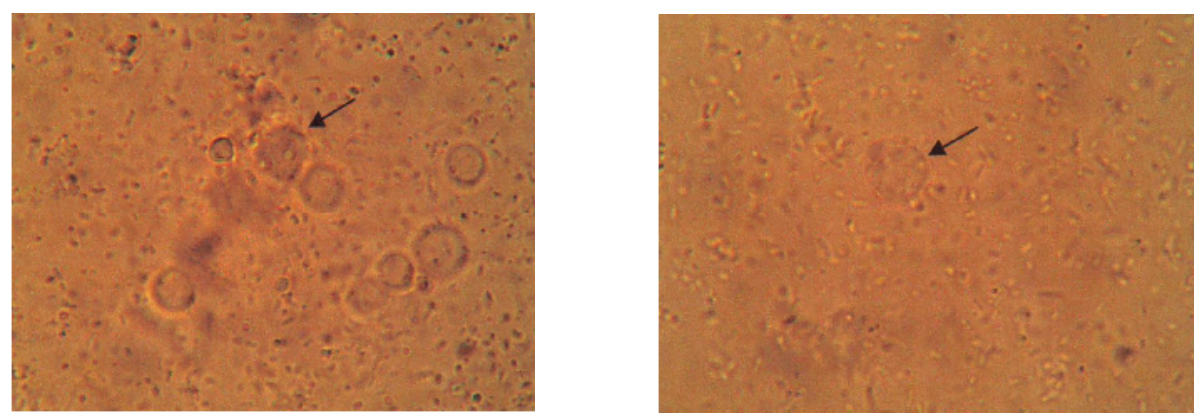

Fig (5): E.histolytica in culture media that treated with EEP showed cyst which consist a large glycogen vacuole and lateral nuclei $(\mathbf{1 0 0 X})$.

\section{Discussion}

Amoebasis is one of the most common nonviral causes of diarrhea among children, Metronidazole is still the most widely used drug for amoebasis treatment, although there are some problems related to resistance and toxicity. Thus, the search for new alternative treatments for amoebasis is necessary, such as natural products.

Propolis is a resinous hive product collected by bees, it is a natural remedy, and may have many antibiotic, antifungal, antiviral and antitumour properties, although reports of allergic reactions are not uncommon, and is relatively non-toxic [12], Propolis have a role as an alternative treatment for chronic vaginal infection [23]. The pharmacologically active molecules in the propolis are flavonoids and phenolic acids and their esters it has been suggested that it's therapeutic activity depends mainly on the presence of flavonoids, volatile oils and aromatic acids, waxes, resins, balms, pollen grains which are a rich source of essential elements such as magnesium, nickel, calcium, iron and zinc [13]. Ethanol extract of propolis (EEP) is the most common; extracts with other solvents have been carried out for identification of more than 200 constituents [12]. Ethanolic extracts of propolis samples showed high antibacterial activity against Gram-positive cocci (Staphylococcus aureus), but had a weak activity against Gram-negative bacteria (Escherichia coli and Pseudomonas aeruginosa) and yeast (Candida albicans) [24], This activity is reported to be due to flavonoids and aromatic acids and esters present in the resin [12], but the relationship between the structure and antibacterial activity of propolis constituents is unknown.

In addition propolis ethanolics have been found to be effective against protozoal, some authors have studied the antiparasite properties of propolis against Trypanosoma cruzi [9, 15, 16, 25], Trichomonas vaginalis [14], Giardia duodenalis r1 71 Tl... th. 
present work was carried out to evaluate the in vitro activity of propolis extract on growth and morphological changes of E. histolytica trophozoites.

Toxoplasma gondii, Trichomonas vaginalis and Entamoeba histolytica, were incubated in vitro with various concentrations of propolis extracts with no activity against E. histolytica. Activity against Toxoplasma gondii and Trichomonas vaginalis was evident only after $24 \mathrm{~h}$ of incubation with propolis extracts at concentrations of $150 \mathrm{mg} / \mathrm{ml}[14,26]$. Another study was carried out aiming to evaluate the in vitro effects of an ethanolic extract of propolis on the growth and adherence of Giardia duodenalis trophozoites.

In fact, propolis containing several constituents that act on the enzymes involved in controlling airway responsiveness, like quercetin that inhibits the lipoxygenase, protein kinase $\mathrm{C}$, cyclic AMP phosphodiesterase and apigenin that inhibit the MAP kinase [9].

Propolis can induce morphological alterations in the parasite, but this aspect needs further investigation, it also showed a marked activity on cell lysis of trophozoite, this could lead to alternative for the chemotherapy of E. histolytica however, in vitro effects of this product on trophozoites have not been reported. Thus, the present work was carried out to evaluate the in vitro activity of propolis extract on growth and morphological change of E. histolytica trophozoites.

\section{References}

1. Espinosa-Cantellano, M. and Martinez-Palomo, A.(2000). Pathogenesis of intestinal amebiasis: From molecules to disease. Clin. Microbiol. Rev., 15:318-331.

2. Stanley, S. L. (2003). Amoebiasis. Lancet, 361:1025-1034.

3. Eichinger, D. (2001). Encystation in parasitic protozoa. Curr. Opin. Microbiol., 4:421426.

4. Cimanga, R.K.; Kambu, K.; Tona, L.; Hermans, N.; Apers, S.; Totte, J.; Pieters, L. and Vlietinck, A.J. (2006). Cytotoxicity and in vitro susceptibility of Entamoeba histolytica to Morinda morindoides leaf extracts and it's isolated constituents. J. Ethnopharmacol., (corrected proof).

5. Upcroft, P. and Upcroft, J.A. (2001). Drug targets and mechanisms of resistance in the anaerobic protozoa. Clin. Microbiol. Rev., 14:150-164.

6. Kusumoto, T.; Miyamoto, R.H.; Doi, S.; Hiroyuki, S.; Yamada, H. (2001). Isolation and structures of two new compounds from the essential oil of Brazilian propolis. Chem. Pharm. Bull., 49, 1207-9.

7. Bosio, K; Avanzini, C.; D'avolio, A.; Ozino, O. and Savoia, D.(2000). In vitro activity of propolis against Streptococcus pyogenes. Letters in Applied Microbiology, 31:174-177.

8. Garcia-Viguera, C. (1992). Composition of propolis from two different Spanish regions. $Z$. Naturforsch., 47: 634-637.

9. Marcucci, M. C.; Ferreres, F.; Garcia-Viguera C.; Bankova V.S.; De Castro, S. L.; Dantas, A. P.; Valente, P. H. M. and Paulino, N. (2001). Phenolic compounds from Brazilian propolis with pharmacological activities. Journal of ethnopharmacology, 74:105-112.

10. Bankova, V.S.; Castro, D.S.L. and Marcucci, M.C. (2000). Propolis: Recent advances in chemistry and plantorigin. Apidologie, 31: 3-15. 
11. Pereira, A.S.; M. Norsell, J.N.; Cardoso and Aquino Neto, F.R. (2000). Rapid screening for polar compounds in Brazillizn propolis by High-Temperature High- Resolution Gas Chromatograpgy-Mass

12. Burdock, G. A.(1998). Review of the Biological Properties and Toxicity of Bee Propolis (Propolis). Food and Chemical Toxicology 36: 347-363.

13. Castaldo, S. and Capassob, F.(2002). Propolis, an old remedy used in modern medicine. Fitoterapia, 73:S1-S6.

14. Starzyk, J.; Scheller, S.; Szaflarski, J.; Moskwa, M. and Stojko, A. (1977). Biological properties and clinical application of propolis. II. Studies on the antiprotozoan activity of ethanol extract of propolis. Arzneimittelforschung. , 27:1198-9.

15. Higashi,K.O.; de Castro, S.L. (1994). Propolis extracts are effective against Trypanosoma cruzi and have an impact on its interaction with host cells. Journal of Ethnopharmacology, 43: 149-155.

16. Prytzyk, E.; Dantas, A. P. ; Salomão, K.; Pereira, A. S.; Bankova, V. S.; De Castro, S. L. and Aquino Neto, F. R.(2003). Flavonoids and trypanocidal activity of Bulgarian propolis. Journal of Ethnopharmacology, 88: 189-193.

17. Freitas,S.F. ; Shinohara, L. ; Sforcin,J.M. and Guimaraes,S. (2006). In vitro effects of propolis on Giardia duodenalis trophozoites. Phytomedicine , $13: 170-175$.

18. Clark, C.G. and Diamond, L.S. (2002). Methods for cultivation of luminal parasitic protists of clinical importance. Clin. Microbiol. Rev., 15:329-341.

19. Miorin ,P.L.; Levy Junior, N.C.; Custodio, A.R.; Bretz, W.A.and Marcucci, M.C. (2003).Antibacterial activity of honey and propolis from Apis mellifera and Tetragonisca angustula against Staphylococcusaureus. J Appl Microbiol., 95: 913-920.

20. Arrais-Silva, W.W.; Colhone, M.C.; Ayres, D.C.; Souto, P.S.and Giorgio, S. (2005). Effects of hyperbaric oxygen on Leishmania amazonensispromastigotes and amastigotes. Parasitol Int., 54: 1-7.

21. Lwin, K.M. and Oo, M. (2004). In vitro anteamoebicidial activity of "Dysenzi" on Entamoeba histolytica in cultures. FAME Pharmaceuticals Co., Ltd. Internet:http:// Famepharma.com.

22. Jaffer,H.L.; Mahmed,M.J.; Jawad,A.M.; Naji,A.; AL-Naib,A. (1983). Phytochemical and Biological screening of some Iraq plant. Fitoterapia .p:299.

23. ImhofT,M.; Lipovac, M. ; Kurz,Ch.; Barta,J.; Verhoeven, H.C. and Huber ,J.C. (2005). Propolis solution for the treatment of chronic vaginitis.International Journal of Gynecology and Obstetrics, 89:127-132.

24. Silici, S. and Kutluca, S. (2005). Chemical composition and antibacterial activity of propolis collected by three different races of honeybees in the same region. Journal of Ethnopharmacology, 99: 69-73.

25. Silva Cunha ,I. B. ; Salomao ,K.; Shimizu ,M.,; Bankova, V. S.;Custodio ,A. R.,; Castro, S. L. and Marcuccle ,M. C. (2004). Antitrypanosomal Activity of Brazilian Propolis from Apis mellifera. Chem. Pharm. Bull. 52: 602-604.

26. Dobrowolski,J. W.; Vohorab, S.B. ; Sharmab,K.; Shahb, S. A.; Naqvib,S.A.H. and Dandiyab, P.C. (1991). Antibacterial, antifungal, antiamoebic, antiinflammatory and antipyretic studies on propolis bee products. Journal of Ethnopharmacology, 35: 77-82. 